\title{
Compromiso con la formación inicial de docentes Clase invertida y TIC
}

\section{Commitment to initial formation of teachers Flipped classroom and ICT}

\author{
Daiana Yamila Rigo ${ }^{1} \&$ Paola Paoloni ${ }^{2}$
}

Fecha de recepción: 30/07/2018; Fecha de revisión: 03/11/2019; Fecha de aceptación: 29/04/2019

Cómo citar este artículo:

Rigo, D. Y. \& Paoloni, P. (2019). Compromiso con la formación inicial de docentes. Clase invertida y TIC. Revista de Innovación y Buenas Prácticas Docentes, 8(4), 102-115.

Autor de Correspondencia: daianarigo@hotmail.com

\section{Resumen:}

Este escrito presenta un estudio realizado con estudiantes universitarios de titulaciones de formación de diversos profesorados de la Universidad Nacional de Río Cuarto, Argentina. Específicamente, se tuvieron como objetivos conocer qué recursos de la comunicación y de la información los estudiantes reconocen y usan en sus prácticas docentes supervisadas de enseñanza como futuros docentes, así como comprender la percepción que tienen del modelo de la clase invertida como metodología a usar en sus futuras clases, como profesionales. Para ello se desarrolló una experiencia de innovación que, grosso modo, consistía en la planificación de una clase invertida, como trabajo práctico integrado en una de las materias del área pedagógica central del plan de estudio en lo que refiere a su formación docente. Para la recolección de datos, se utilizaron dos cuestionarios con preguntas abiertas. El análisis de datos cualitativo se realizó en función del enfoque interpretativo, mostrando como principales resultados que las TIC están casi ausentes en las prácticas de enseñanza de grado y que los potenciales profesores reconocen como prometedor el modelo de la clase invertida para desarrollar sus clases de cara al posterior ejercicio del rol.

Palabras clave: clase invertida, compromiso, formación de formadores, universidad.

\begin{abstract}
:
The paper presents a study carried out with university students of different professors from the National University of Río Cuarto, Argentina. Specifically, the objectives were to know what communication and information resources recognize for the students and use in their supervised teaching practices as future teachers, as well as to understand their perception of the model of the flipped classroom as a methodology to be used in future classes, as professionals. For this, an experience of innovation was developed which, in a broad way, consisted in the planning of a flipped classroom as practical work integrated in one of the subjects of the central pedagogical area of the study plan in what refers to their teacher training. For data collection, two questionnaires with open questions were used. The qualitative data analysis was carried out according to the interpretative approach, showing as main results that ICT are almost absent in the teaching practices of degree and that potential teachers recognize as promising the model of the flipped develop their face classes to the subsequent exercise of the role.
\end{abstract}

Key Words: flipped classroom, commitment, training of trainers, university.

\footnotetext{
1 Universidad Nacional de Río Cuarto - Consejo Nacional de Investigaciones Científicas y Técnicas (Argentina), daianarigo@hotmail.com ; CÓDIGO ORCID: https://orcid.org/0000-0003-0312-6429?lang=es 2 Universidad Nacional de Río Cuarto - Consejo Nacional de Investigaciones Científicas y Técnicas (Argentina), paopaoloni17@hotmail.com ; CÓDIGO ORCID: https://orcid.org/0000-0002-9384-010X
} 


\section{INTRODUCCIÓN}

El desafío de formar docentes en el siglo XXI se vincula a la necesidad de generar espacios formativos en torno a los conocimientos establecidos en la Psicología de la Educación, para el desarrollo de propuestas de innovación educativa en torno a la incorporación de las Tecnologías de la Información y la Comunicación -TIC-, que han aportado cambios a los modelos de formación tradicionales, dotando de herramientas que han favorecido la autonomía del alumno y diversificado el rol del formador. No obstante, se vislumbra que el compromiso con las TIC en los planes de estudio es aún escaso y su incorporación en los programas de formación de futuros profesores se produce lentamente, en tanto los programas de las materias concernientes a las áreas de formación pedagógica no se han revisado conjuntamente con el avance registrado en la sociedad de la información (Martín Cuadrado, 2010).

En tal sentido, el compromiso con la formación docente y las TIC, deviene en un doble propósito, primero en la necesidad de formular cambios en los procesos de enseñanza y aprendizaje de los espacios formativos en las titulaciones de formación de profesorados que se dictan al interior de las universidades, incorporando modelos pedagógicos híbridos como herramientas para el futuro ejercicio de la docencia; y segundo, un compromiso que se desplaza en la posibilidad de implicar a sus futuros estudiantes con recursos y modelos pedagógicos que combinen los contextos tradicionales con el uso de entornos virtuales de formación, enseñanza y aprendizaje. Tal como lo exponen Hernández Silvaa \& Tecpan Flores (2017), la necesidad de incorporar las TIC se acrecienta a medida que la brecha entre los modelos educativos tradicionales y las necesidades de los estudiantes 'conectados' se ensancha; son los programas de formación docente los que deben adaptarse a este contexto, ya que los nuevos profesores basan sus prácticas docentes en gran medida, en el conocimiento y habilidades aprendidas en su formación inicial.

En este marco, y con la intención de actualizar la formación inicial de docentes, desarrollamos una investigación dentro del espacio de titulación formación de diversos profesorados de la Universidad Nacional de Río Cuarto, incorporando dentro del programa de la materia Elementos de la Psicología Educacional, en la unidad 4, contenidos para configurar entornos favorables para el aprendizaje, 'TIC y contextos académicos. Nuevos recursos para la formación de formadores', a modo de ofrecer un nuevo modelo de enseñanza y aprendizaje basado en el enfoque de la clase invertida.

Específicamente, el estudio desarrollado se propuso profundizar sobre dos objetivos, en primera instancia, explorar qué conocimientos tienen los estudiantes de distintas titulaciones de formación de profesorados sobre las TIC, como recursos integrados en su formación actual; y en segunda instancia, describir y comprender la percepción que alumnos del profesorado sustentan sobre el modelo de clase invertida en relación con sus futuras prácticas como docentes.

\subsection{Formación de futuros docentes y el modelo de la clase invertida}

La necesidad de que la formación del profesorado se ajuste a los nuevos requerimientos de la sociedad de la información, caracterizada por conocimientos diversos y globales, donde Internet y la era digital han impactado en la forma de acceder y difundir el saber; deviene de una concepción de la enseñanza que ha dejado de ser exclusividad de los docentes, y de un aprendizaje que lejos de ser una actividad interna e individual, se comprende como una actividad colectiva, en línea y disponible en la nube. 
Prensky (2010) considera que generar una verdadera innovación en el aula dependerá de cómo ésta sea diseñada, el objetivo esencial debe orientarse a enseñar a usar los nuevos modelos pedagógicos en torno a las TIC, en lugar de digitalizar las aulas. Lo cual supone que la educación debe sufrir un cambio, en la que el profesor ya no sea visto como el centro del proceso formativo; donde la enseñanza esté centrada en el estudiante, basada en la colaboración entre pares y en la participación activa.

Aunque el escenario educativo que predomina es más afín al estilo magistral de clases expositivas donde el estudiante tiene un rol mayormente pasivo (Gaete Quezada, 2011), ligados a planes de estudios que muestran ausencia de contenidos que aborden nuevos paradigmas y modelos educativos; el reto, en tal sentido, es transformar estos últimos para impactar sobre la formación inicial y en el posterior ejercicio profesional.

Díaz (2010), propone que el reto de innovar en el campo de las instituciones de educación superior la formación de los profesores, para mejorar la calidad de sus prácticas docentes debe incorporar los recursos tecnológicos como pieza relevante. Prácticas que, sustentadas en actividades de aprendizaje que tienden a promover la memorización y atomización del saber (Escontrela Mao, 2008; Nouri, 2016), necesitan una modificación de cara a las TIC.

Lo que supone encaminar trasformaciones e innovaciones educativas que tiendan a superar estos modelos de prácticas tradicionales de contenidos estructurados, de la mano de la implementación de las TIC en la formación inicial de docentes. En este contexto de cambio, surge como alternativa el modelo llamado Aula Invertida, o también conocido como Flipped Classroom, para mejorar los procesos de enseñanza y aprendizaje. Específicamente, Uzunboylu \& Damla (2017) analizan las tendencias de los últimos años, relacionados con la incorporación de las TIC en el entorno educativo, y advierten que el modelo muestra muchas promesas. Lo integran dentro de las metodologías denominadas como el blended learning o aprendizaje mixto, el cual tiene sesiones presenciales y otras virtuales, desde la cual se promueve la interactividad y la motivación a partir del trabajo colaborativo de los estudiantes y docentes, favoreciendo el aprendizaje autónomo y autorregulado (González, Perdomo \& Pascuas, 2017).

Aguilera Ruiz, Manzano León, Martínez Moreno, Lozano Segura \& Yanicellies (2017), indican que es un método de enseñanza cuyo principal propósito es que los estudiantes asuman un rol mucho más activo en su proceso de aprendizaje en comparación con el que venían ocupando tradicionalmente. El concepto del aula invertida o volteada, es un modelo de aprendizaje donde la enseñanza directa es sustituida por videos, y durante las clases, se propone a los estudiantes enfocar su atención en actividades de aprendizaje importantes, guiados por los docentes y resueltas de manera grupal entre los estudiantes.

En definitiva, supone mover la 'entrega' del material fuera de la clase formal y hacer uso de ésta para emprender actividades colaborativas. Es decir, se busca integrar las nuevas tecnologías en la enseñanza de contenidos utilizando activamente materiales audiovisuales, combinado videos, resúmenes de conferencias, animaciones o tutoriales (Bergmann \& Sams, 2012; Martín Rodríguez \& Santiago Campión, 2016; Opazo Faundez, Acuña Bastias \& Rojas Polanco, 2016; Rigo \& Paoloni, 2017; Ros Gálvez \& Rosa García, 2014).

Asimismo, Blasco, Lorenzoi \& Sarsa (2018), exponen que no sólo se invierten los momentos tradicionales de la clase, sino que también se transforman los roles y los perfiles de los profesores -de experto a colaborador- y de los estudiantes -de oyente pasivo a constructor del conocimiento-, bajo una interacción dialéctica centrada en el alumno. Proceso que posiciona al modelo de clase invertida dentro del enfoque socioconstructivo y del conectivismo como paradigma emergente en la sociedad del conocimiento. Es decir, tal como lo expresan Simon Llovet, Ojando Pons, Avila Morena, Miralpeix Bosch, Lopez Vicente \& Prats Fernández (2018), las teorías del aprendizaje se han adaptado a los entornos cambiantes, en una evolución que va de la construcción del conocimiento (constructivismo), a un aprendizaje en sociedad (socioconstructivismo), a un estadio actual definido por la co-construcciòn de los saberes 
(conectivismo), que se explica por el creciente uso de las redes sociales y los intercambios que promueven un aprendizaje auto-gestionado y colaborativo, destacándose el compromiso activo para compartir información y conocimiento. Precisamente esta trayectoria seguida por el modelo de la clase invertida genera un contexto propicio para para incluirlo dentro de la formación de futuros formadores.

\section{METODOLOGÍA}

Se llevó a cabo un estudio exploratorio-descriptivo de corte cualitativo, con el objetivo de explorar qué recursos en torno a las TIC usan los profesores formadores de futuros docentes en sus espacios curriculares y, describir y comprender la percepción de los alumnos de diversas titulaciones de formación de profesorados sobre el modelo de clase invertida como alternativa pedagógica potencialmente útil para su futuro desempeño docentes. De la innovación participaron todos los estudiantes matriculados en la materia Elementos de la Psicología Educacional $(n=51)$, dictada en la UNRC-Argentina, durante el primer cuatrimestre del 2018. Específicamente, la muestra estuvo constituida por alumnos de tres titulaciones de formación de profesorados, a saber: Lengua y Literatura, Historia y Matemática. Cabe aclarar, que la materia es obligatoria en las diferentes titulaciones y está ubicada en el plan de estudio de profesorados pertenecientes a la Facultad de Ciencias Humanas y a la Facultad de Ciencias Exactas Físico-Químicas y Naturales de la UNRC; específicamente en el segundo año de los Profesorados de Lengua y Literatura y Matemáticas por Humanas, y en el tercer año del profesorado de Matemática por Ciencias Exactas.

\subsection{Experiencia de innovación desarrollada}

Como se dijo, esta experiencia de innovación se desarrolló en el marco del programa de Elementos de la Psicología Educacional, particularmente dentro de la unidad 4, denominada 'Sobre los contextos favorables para el aprendizaje', punto 4.5 'Tic y contextos académicos. Nuevos recursos para la formación de formadores'. La incorporación del contenido tuvo como finalidad ofrecer a los estudiantes conocimientos sobre cómo integrar las TIC a sus futuras prácticas docentes, siendo este espacio curricular central en los planes de estudio de los profesorados, en lo que refiere estrictamente a la formación pedagógica.

Se desarrolló durante tres semanas, con dos encuentros presenciales. El primero se planificó específicamente para presentar teóricamente el modelo de clase invertida y la actividad académica práctica que los estudiantes tenían que desarrollar en torno al modelo pedagógico desarrollado.

Para la presentación de las características del modelo se usaron diapositivas que lo definían, remarcando los puntos favorables y las desventajas que la bibliográfica actual expone sobre la clase invertida. Consecutivamente, se les explicó el trabajo práctico que debían desarrollar en grupos de no más de cinco integrantes y agrupados según la titulación de formación de profesorado de pertenencia: Lengua y Literatura, Matemática o Historia.

A cada grupo se le solicitó la siguiente consigna:

Planificar una clase invertida seleccionado un tema que a futuro sea objeto de enseñanza en su rol como docentes, explicitando el por qué utilizarían la metodológica para sus clases. En primera instancia, deberán delimitar a quienes está dirigida la clase -estudiantes de nivel secundario o universitario-. En segunda instancia deberán describir y señalar qué recursos (videos, link, páginas web, etc.) usarían para presentar el tema a los estudiantes, cómo guiarían la actividad, qué dispositivos usarían como 
disparadores de participación y debate en torno a los contenidos enseñados. Asimismo, explicitar en qué tiempo y en qué plataforma virtual desarrollarían la clase invertida (aula virtual, Facebook, Blogs, etc.). En tercera instancia, tendrán que presentar la actividad práctica que los estudiantes deberían resolver en la clase presencial a modo de poner el conocimiento en acción y favorecer la transferencia y la comprensión de los contenidos centrales trabajados fuera de la clase. Asimismo, deben indicar cómo iniciarán la clase en el aula para chequear si hay dudas o dificultades, sin caer una mera exposición.

Las dudas e intercambios se canalizaron en un grupo de cerrado de Facebook creado con anterioridad por la docente responsable de la materia. La misma red se utilizó como canal para compartir experiencias previas desarrolladas conforme el modelo de clase invertida, así como material de consulta y videos explicativos que servían para profundizar los detalles a tener presente a la hora de esbozar la planificación, tales como, el sitio web disponible en https://www.theflippedclassroom.es/ o los videos compartidos en https://elpais.com/.../10/28/actualidad/1477665688_677056.html.

Finalmente, los estudiantes en el segundo encuentro presencial, expusieron sus producciones e intercambiaron opiniones y alternativas con el resto de sus compañeros y los docentes.

\subsection{Instrumentos utilizados}

La recolección de datos se realizó en dos etapas, contando con el consentimiento informado de todos los estudiantes. En la primera se administró un cuestionario ad hoc, conformado por preguntas abiertas, antes de iniciar el desarrollo del primer encuentro presencial. Se usó con la finalidad de recabar información acerca del uso de las TIC que hacen los docentes universitarios en el dictado de las clases teóricas y prácticas, la formación sobre TIC que han recibido en su formación actual de grado, y el uso de éstas en sus primeras aproximaciones a la práctica profesional, así como, si conocían el modelo de clase invertida o qué podían evocar acerca de su denominación. Las preguntas incluidas fueron:

Respecto a tu educación actual, como futuro profesor, ¿qué formación sobre las TIC has recibido?; En las clases teóricas-prácticas, ¿qué recursos de la comunicación y de la información usan los docentes universitarios?; En los primeros acercamientos a tu práctica docente, ¿Has utilizado alguna TIC? Sí-No, ¿Por qué tomaste la decisión de incorporarlas a tu clase?; y ¿Conoces el modelo de la clase invertida? Sí-No, Podrías ofrecer una breve descripción sobre lo que su denominación podría significar.

En la segunda etapa, posterior a la experiencia desarrollada, los estudiantes completaron un nuevo cuestionario ad hoc, integrado también por preguntas abiertas; que consultaban acerca de la percepción que tenían sobre el modelo de la clase invertida para renovar las prácticas de enseñanza y aprendizaje, resultando sus ventajas y desventajas como recurso para la enseñanza y los aprendizajes de los alumnos. Específicamente, las preguntas fueron:

¿Cuál es tu opinión respecto al modelo de clase invertida para renovar las prácticas de enseñanza y aprendizaje? Nombra ventajas y desventajas; ¿Cómo futuro docente, incluirías en tus clases el modelo de Clase Invertida? Sí-NO ¿por qué (pensando en la enseñanza) y para qué (pensando en el alumno) ?; ¿Cómo te percibiste durante la realización del práctico, respecto a participación e interés?; y ¿Consideras que el modelo puede "enganchar a los estudiantes" con sus procesos de aprendizaje?, ¿Por qué? 


\section{RESULTADOS}

Los resultados que a continuación se sintetizan se derivan de un análisis interpretativo, siguiendo las pautas recomendadas por Vasilachis (2007), del cual emergen dos categorías correspondientes a cada instancia de recolección de datos que permiten tener una apreciación de los participantes con respecto a la experiencia de innovación desarrollada.

\subsection{Hacia las TIC...Camino poco transitado}

Las respuestas de los estudiantes al primer cuestionario, muestran por un lado que las TIC en la formación docente actual es un contenido más ausente que presente en los programas y planes de estudio de grado. La mayoría alude que muy pocas veces se trabaja como contenido en el marco de una materia; no obstante, algunos remarcan que los docentes hacen comentarios aislados respecto a su potencial uso para mejorar el aprendizaje y la motivación de los estudiantes.

"Menciones en clase, breves, pero no de forma específica" (YV).

"Actualmente en la carrera nunca trabajamos con las TIC como objeto de estudio propiamente dicho" (TJ).

"Ninguna hasta el momento. Sólo sé que las TIC son las Tecnologías de la información y de la comunicación. También las hemos nombrado en algunas asignaturas, de la importancia de implementarlas en las clases, etc." (FC).

"Con respecto a mi educación actual, no tuve mucha formación. Si en las distintas materias hemos visto como a veces repercuten las TIC en el aula, pero no como trabajar con ellas y los alumnos hasta este momento" (CG).

"El único acercamiento qué tuve con las TIC, hasta el momento son las que nos han proporcionado desde esta materia" (JB).

"He recibido escasa información sobre las TIC, de hecho, creo que debería hacerse más hincapié en esta cuestión, que es algo que nos atañe actualmente como futuros profesores" $(A B)$.

"La verdad que ninguna, lo poco que se sobre TIC lo he leído por mi parte" $(A D)$.

"He recibido, a lo largo de la carrera poca información acerca de qué son exactamente las TIC. Si debo destacar que como futura docente me interesé en el tema y amplié mis conocimientos acerca de ésta a través del amplio mundo del internet; que hacen a la misma" (NL).

Por otro lado, esta ausencia en la formación inicial de grado se transfiere en sus primeros acercamientos a las prácticas docentes supervisadas solicitadas como parte de sus procesos de formación. Los alumnos expresan no usar las TIC, aludiendo en su mayoría a la falta de conocimiento que tienen al respecto:

“En la práctica que realicé no consideré incorporar las TIC" (ZG). 
"No contaba con la formación, ni los recursos necesarios para implementarlas" (BL).

"No sabría cómo poder incorporar las TIC a las clases de matemática" (PV).

"No he utilizado ninguna TIC en las prácticas que he dado...solo utilice fotocopias" (AB).

"En las prácticas socio comunitarias, pero en ellas no utilizamos las TIC por el momento porque no sabíamos cómo incorporarlas" (CG).

Y si lo hacen, los recursos utilizados son los que predominan en la clase de sus formadores, fundamentalmente las presentaciones con diapositivas, así lo mencionaban: "En la primera práctica de la carrera utilice Power Point" (SA).

En tanto cuando se les consulta sobre el uso que hacen los docentes universitarios en las clases teóricas y prácticas, los estudiantes manifiestan que las TIC se hacen un poco más visible. Reconocen que los profesores usan recursos o dispositivos para dictar la clase o exponer un tema, acotados a las presentaciones y videos, y una fuerte presencia de las fotocopias, la tiza y el pizarrón como principales soportes:

"Videos o Power Point" (CG).

"Los docentes universitarios utilizan material teórico fotocopiado" (MG).

"Proyección de contenidos durante la clase a través del mono cañón" (MF).

"Pizarrón y tiza" (JP).

"Explicaciones llevada a cabo en el pizarrón" (RM).

"Algunos docentes usan un cañón y pasan Power Point o videos en algunas clases. Otros simplemente utilizan el pizarrón y nada más que eso" $(\mathrm{CA})$.

"Con respecto a las clases nos proyectan Power Point, algunas profes nos suben PDF sobre las copias y demás" (JB).

Sin embargo, a pesar del uso infrecuente y el limitado listado de recursos asociados a la tecnología de la información y de comunicación que se utilizan en clases, aparece como fuerte la idea de renovar las prácticas educativas, en tanto los estudiantes perciben que la falta de formación, hace más difícil su inclusión en sus prácticas actuales y posteriores.

"Creo que esta forma de utilizar la tecnología me da herramientas para poder abordar una futura clase de una manera diferente, nos acercan posibilidades de atraer a los alumnos a que presten mayor atención probablemente $(A B)$.

"Creo que tener una formación sobre las TIC es muy importante en la actualidad; lamentablemente en este aspecto hay que ser un autodidacta" (MD).

"Es importantísimo, ya que actualmente estamos invadidos por las nuevas tecnologías, los jóvenes utilizan mucho las redes sociales, el uso del celular es un instrumento que debe incluirse en las clases y debe plantearse los nuevos rumbos de cómo dar las materias. Hoy en da la información está a un clic, solo falta enseñar a formar estudiantes críticos para saber cómo utilizar la cantidad de informar que circula" (RM). 
"Falta formación para poder usarlas el día de mañana" (MG).

"Tendremos alumnos a los que no podremos ayudar por falta de conocimiento. Ello implica un ajuste en nuestra formación" (MA).

Asimismo, aparecen algunos beneficios que los futuros formadores vinculan al uso de las TIC hacia los procesos de enseñanza y aprendizaje de los estudiantes, tales como la promoción de la motivación, el compromiso, la posibilidad de establecer aprendizajes más significativo ligados a una comprensión más profunda, con la posibilidad de acceder al conocimiento a partir de la web y diversas fuentes, logrando un mayor acercamiento a las nuevas generaciones de adolescentes, quienes tienen una gran afinidad con las tecnologías.

"Sobre todo porque influye fuertemente en el aprendizaje de los estudiantes, se muestran más entusiasmados a través de las TIC y para los docentes es un nuevo desafío" (PS).

"Usar tecnología como recurso de comunicación permite que los alumnos exploren en las redes y puedan construir conocimientos" (JP).

"Son herramientas que pueden ayudar a la clase, a estar más atentos 0 generar un interés más grande por la materia" (EB).

"Tener un buen acercamiento a los niños/adolescentes que hoy en día utilizan tecnología" (PV).

"Permiten entender mejor un concepto o un tema en especial" (AL).

"Ayudan a la motivación, innovan llama la atención y se convierten en situaciones de aprendizaje recordables" (PM).

Por último, encontramos que la representación que se hacen del modelo de clase invertida, sólo se liga a un cambio de rol, donde los estudiantes asumen las tareas de los docentes, preparan y dan la clase, sin mencionar el uso de las TIC en ese cambio de actividades.

"Creo que el modelo de la clase invertida se basaría en una clase donde se inviertan los roles del profesor-alumno. Donde el alumno daría una clase, explicando algún tema de su conocimiento y el profesor aprenda sobre eso" (FC).

"Por el nombre que tiene, se me ocurre pensar en que es una clase en dónde invierten los roles alumnos con profesores, siendo una clase abordada por alumnos y escuchada por profesores" (TF).

"Supongo que una clase invertida se trataría de que el alumno prepare, planifique, enseñe, y dicte una clase de un tema específico a otros alumnos e incluso al profesor. En este último, se podría hablar de que la clase se ha invertido, haciendo que el alumno se convierta en el profesor y este mismo en alumno" (BJ).

"Supongo que tiene que ver con invertir los papeles del docente y el alumno" (AL). 
"Supongo que sería cambiar los roles con los alumnos y que los alumnos sean los que preparan la clase y el profesor escucha la clase que dan y en base a eso evalúa o guía a los alumnos" (JJ).

"Para mi significa ponernos en el lugar de los profes y dictar una clase a nuestra manera" (JB).

En síntesis, las voces de los estudiantes muestran con claridad que la inclusión de las TIC en los planes de estudio es aún en el siglo XXI una materia pendiente; que comprendidas por los estudiantes como recursos imprescindibles para renovar e innovar su formación y posterior ejercicio profesional, insta a tomar como meta prioritaria dentro de la agenda educativa al interior de los profesorados la revisión de los programas y los contenidos de las materias que conforman el núcleo de formación pedagógica.

\subsection{Compromiso con la formación...hacia modelos pedagógicos mixtos}

La propuesta inserta en el programa de la materia Elementos de la Piscología Educacional dictada a estudiantes matriculados en diversos profesorados, generó apreciaciones positivas sobre las implicancias hacia el futuro rol como docentes. En principio, se destaca que el modelo de clase invertida, los movió del lugar común de hacer docencia a partir de clases expositivas hacia un nuevo rol donde la planificación cobra centralidad para promover la participación de los estudiantes a partir de la inclusión de las TIC.

"Me resultó interesante porque nos incentiva a pensar y a crear una nueva manera de enseñar utilizando las TIC, lo que nunca antes había hecho" (JK).

"Realizar una actividad, que realizaremos como futuros profesionales" (BP).

"Fue desafiante y me sentí predispuesta para aprender a enseñar de otro modo" (AM).

"Me pareció una propuesta distinta, hace rato pensábamos trabajar con la idea de audio cuentos, y creo que la clase invertida fue un modelo perfecto para eso" (YT).

"Fue una experiencia interesante, en el sentido de que al desconocer esta modalidad o no saber, al involucrarme en la planificación tomó una significación positiva" (YV).

"Me pareció interesante planificar una clase con esta nueva metodología" $(\mathrm{CA})$.

"Me gustó mucho crear una clase diferente a lo que generalmente usamos en la carrera" (Al).

"Me motivó y me desafió porque me gusta trabajar con lo que no es tan cotidiano, porque todo constituye un aprendizaje" (GB).

"Me resultó interesante, porque como futura docente quiero que mis alumnos aprendan de manera participativa y no sólo escuchen una explicación" (AL).

Asimismo, entre los comentarios de los estudiantes que participaron de la experiencia se destacan algunos aspectos que rescatan del modelo, aludiendo al impacto que éste puede tener sobre el compromiso de los estudiantes, mencionando 
mayor interés, participación, interacción, a la vez de autonomía y autorregulación, en tanto la clase ha sido planificada y andamiada por el docente.

"Me parece muy útil para acelerar las clases y aprovechar mejor el tiempo en el aula, además incluir las TIC hace que las prácticas educativas sean más participativas" (EB)

"Mejor administración del tiempo y más interesante" (ML)

"Buen modelo porque incorpora recursos audiovisuales o las redes sociales, los alumnos se incentivan a realizar las tareas, mayor interacción entre alumnos y docentes. A la vez incentivo a que el alumno se vaya convirtiendo en autónomo" (AJ)

"Se adquiere mayor autonomía y control, manejar sus tiempos, puede consultar varias fuentes, no solo el dado por el docente, sino también hacer más investigaciones" (RM).

"Muy buena propuesta, o modelo pedagógico para despertar a los chicos u sacarlos de la 'zona durmiente' que provoca la clase tradicional" (AM).

"Creo que no sólo depende del modelo sino del docente y la manera en que se trabaja con los alumnos, por eso estuvo bueno conocer el modelo y luego planificar una clase invertida" (IJ).

"Refiere mayor esfuerzo, pero me gustó mucho" (DR).

"Los alumnos se vuelven más autorregulados y metacognitivos" (GG).

"Motiva y genera aprendizajes significativos, sólo si hay una planificación minuciosa" (GG).

Agregan como consideraciones significativas del modelo, la conexión que puede lograse con los adolescentes, sus potenciales alumnos, que guardan un vínculo estrecho con las TIC. En tal sentido, rescatan que incorporar modelos mixtos a la enseñanza, ayuda a producir un acercamiento a los estudiantes que, inmersos en la sociedad de la información, son consumidores activos de las tecnologías, y desde ese lugar se puede construir interacciones más dinámicas entre docente y alumnos.

"A los estudiantes les gusta aprender más mirando que escuchando, se conectan más con el tema a aprender" (EB)

"Considero que es un modelo muy coherente a los tiempos actuales y sin duda novedoso, por estas razones creo que es muy factible que funcione como recurso didáctico, útil para la construcción de conocimientos" (MF).

"Es muy motivador para los estudiantes, puesto que está relacionado a lo que tienen en la mano todo el día (celular, computadora) y genera autonomía y control" (VP).

"Los alumnos podrían acceder las veces que les resulten necesarias a los contenidos o explicaciones, videos, etc. hasta lograr comprender a su ritmo 
determinados contenidos, son chicos que están constantemente con los celulares o la compu" (FC).

"Se crea una relación más cercana entre el docente y el alumno" (CA).

"Hoy en día el Internet es lo más usado, lo que ha 'invadido' a todos y todos lo usan, por eso tener al alcance del celular o computadora una clase que normalmente se da en el aula puede ser muy interesante para ellos, son ciberlectores y saben usar aplicaciones y les gusta" (AI).

No obstante, perciben que la desventaja del modelo puede estar asociada a problemas de conectividad, ya sea en ausencia de Internet en los hogares o mala conexión que enlentezca la reproducción o descarga del contenido compartido fuera del aula.

"No todos poseen acceso a Intranet" (EB).

"Falta de infraestructura en las universidades" (ML).

"que algún alumno no se anime a participar" (SJ).

"Hoy en día, si bien la mayoría cuenta con acceso a Internet no toso en su totalidad tienen acceso a una velocidad que permita realizar la actividad propuesta" (RM).

En síntesis, los estudiantes del estudio valoran la inclusión de las TIC en su formación de grado, rescatan tanto aspectos negativos como positivos de la implementación de una clase invertida, que perciben como un modelo prometedor de sus futuras prácticas docentes teniendo como propósito que los estudiantes se vinculen con el saber desde propuestas innovadoras y ligadas a sus intereses por las tecnologías.

\section{CONSIDERACIONES FINALES}

La presente experiencia de innovación se propuso como objetivo indagar la formación que estudiantes de grado de diversos profesorados de la UNRC tenían respecto a las TIC e incluir como contenido de una materia del área pedagógica las tecnologías como aporte a la pedagogía, a partir de una actividad práctica que suponía planificar una clase invertida.

Asumimos ese compromiso con la formación inicial de docentes para dar respuesta a la evolución de la sociedad de la información que paulatinamente debe incorporarse a las teorías educativas a partir de las cuales los docentes diseñan sus clases y los estudiantes aprenden (Catani, 2012). En este marco, el modelo de clase invertida, además de ser un recurso actualizado y en sintonía con la enseñanza y la docencia del siglo XXI, puede ser considerado como un recurso valioso para promover cambios e innovaciones de las metodologías instructivas tradicionales. 
De los resultados, se derivan dos conclusiones interesantes. En primer lugar, la escasa presencia de las TIC en la formación de grado, específicamente en las clases configuradas por los docentes universitarios formadores de futuros profesores. Esta escasa presencia parece corresponderse con la posterior ausencia de las tecnologías en los primeros acercamientos de los alumnos a la práctica profesional, donde la falta de información y conocimiento acerca de cómo hacer un uso pedagógico de las TIC son la pieza clave, a pesar de reconocer sus beneficios sobre la motivación y la construcción del conocimiento.

En segundo lugar, de la experiencia desarrollada en torno a la planificación de una clase invertida como instancia de formación en TIC dentro del programa de estudio de las tres titulaciones de formación de profesorados, muestra ser un escenario propicio, desde la perspectiva de los participantes, para generar instancias de aprendizaje acerca de la inclusión de las tecnologías digitales al mundo del aula tradicional, con impacto en el compromiso con la formación actual y posterior de los estudiantes. Lo cual guarda consonancia con estudios previos que indican la importancia de invertir el aula para favorecer la implicación de los alumnos y a la vez renovar las prácticas instructivas (Cronhjort, Filipsson \& Weurlander 2017; García Gómez, 2016; Hutchings \& Quinney, 2015; James, Chin \& Williams, 2014; Riccetti \& Rigo, 2018; Rigo \& Paoloni, 2017).

Los planteos referidos, ponen sobre el tapete la necesidad de transitar de las TIC a las TAC -Tecnologías del Aprendizaje y el Conocimiento-, en el sentido de lo expresado por Casablancas (2014) de integrar las Tecnologías con sentido pedagógico, propiciando su paso al Aprendizaje y al Conocimiento; hacerlas propias y ubicarlas dentro de aquellas actividades que constituyen y tiñen los procesos educativos, de enseñanza y de aprendizaje.

Por eso la importancia de planificar una clase invertida como instancia de formación inicial, ayuda a construir conocimientos y propiciar aprendizajes significativos con usos tecnológicos, que devendrán en prácticas docentes renovadas, donde los estudiantes puedan aprender el contenido fuera de la clase y luego en ella trabajar colaborativamente, donde el docente sea una guía que apoya y ofrece retroalimentaciones que permiten una dinámica más participaba en la co-construcción del saber. Todo lo expuesto es factible de lograrse si la enseñanza desde la formación de grado genera propuestas pedagógicas valiosas y significativas para sus estudiantes, en un contexto mediado por las TIC y las TAC.

\section{REFERENCIAS}

Aguilera Ruiz, C., Manzano León, A., Martínez Moreno, I., Lozano Segura, M. \& Yanicellies, C. (2017). El modelo flipped classroom. International Journal of Developmental and Educational Psychology, 4 (1), 261-266. Recuperado de: http://www.redalyc.org/articulo.oa?id=349853537027

Bergmann, J. \& Sams, A. (2012). Flip your classroom: Reach every student in every class every day. Oregon: International Society for Technology in Education. Recuperado de:

https://www.liceopalmieri.gov.it/wp-content/uploads/2016/11/Flip-YourClassroom.pdf

Blasco, A. C., Lorenzo, J. \& Sarsa, J. (2016). La clase invertida y el uso de vídeos de software educativo en la formación inicial del profesorado. Estudio cualitativo. 
@tic. Revista D'innovació Educativa, 17, 12-20. Recuperado de: http://www.redalyc.org/jatsRepo/3495/349551247003/349551247003.pdf

Casablancas, S. (2014). De las TIC a las TAC, un cambio significativo en el proceso educativo con tecnologías. Virtualidad, Educación y Ciencia, 5 (9): 106-109. Recuperado de https://revistas.unc.edu.ar/index.php/vesc/article/view/9926

Catani, M. L. (2012). El desafío de formar a formadores. Universidad Nacional de La Pampa. Recuperado de:

http://sedici.unlp.edu.ar/bitstream/handle/10915/42770/Documento_completo.p df?sequence $=1$

Cronhjort, M., Filipsson, L. \& Weurlander, M. (2017). Improved engagement and learning in flipped-classroom calculus. Teaching Mathematics and its Applications: An International Journal of the IMA, hrx007. DOI: https://doi.org/10.1093/teamat/hrx007

Díaz, O. (2010). Formación tecnopedagógica: DIY para tecnófobos. Apertura, 2 (2). Recuperado de: http://www.redalyc.org/articulo.oa?id=68820827011

Escontrela Mao, R. (2008). Hacia un modelo integrador en el uso de las TIC en la educación a distancia. Apuntes y comentarios desde la investigación y la experiencia. Revista de Investigación, 65, 15-31.

Gaete Quezada, R. (2011). La responsabilidad social universitaria como desafío para la gestión estratégica de la Educación Superior: el caso de España. Revista de Educación, 355, 109-133. Recuperado de: http://www.revistaeducacion.educacion.es/re355/re355_05.pdf

García Gómez, A. (2016). Aprendizaje inverso y motivación en el aula universitaria. Revista Pulso, 39, 199-218.

González, M.A., Perdomo, K.V. \&Pascuas, Y. (2017) Aplicación de las TIC en modelos educativos blended learning: una revisión sistemática de literatura. Sophia, 13 (1): 144-154. DOI: http://dx.doi.org/10.18634/sophiaj.13v.1i.364

Hernández Silvaa, C. \& Tecpan Flores, S. (2017). Aula invertida mediada por el uso de plataformas virtuales: un estudio de caso en la formación de profesores de física.

Estudios Pedagógicos, XLIII (3), 193-204. Recuperado de: http://www.redalyc.org/articulo.oa?id=173554750011

Hutchings, M. \& Quinney, A. (2015). The Flipped Classroom Disruptive Pedagogies Enabling Technologies and Wicked Problems Responding to the Bomb in the Basement. Electronic Journal of e-Learning, 13(2), 106-119.

James, A., Chin, C. \& Williams, B. (2014). Using the flipped classroom to improve student engagement and to prepare graduates to meet maritime industry requirements: a focus on maritime education. WMU $J$ Marit Affairs, 13, 331. DOI: 10.1007/s13437-014-0070-0

Martín Cuadrado, A. (2010). Aprendizaje significativo a través de las TIC. Revista Investigaciones en Educación, X (1): 13-35.

Martín Rodríguez, D. \& Santiago Campión, R. (2016) Flipped Learning en la formación del profesorado de secundaria y bachillerato. Formación para el cambio. Contextos Educativos. Número extraordinario, 117-134. DOI: $10.18172 /$ con.2854

Nouri, J. (2016). The flipped classroom: for active, effective and increased learningespecially for low achievers. International Journal of Educational Technology in Higher Education, 13. Recuperado de:

http://www.redalyc.org/articulo.oa?id=501550294006

Opazo Faundez, A., Acuña Bastias, J. \& Rojas Polanco, M. (2016). Evaluación de metodología flipped classroom: primera experiencia. Innoeduca. International. Journal of Technology and Educational Innovation, 2(2), 90-99.

Prensky, M. (2010). Nativos e Inmigrantes Digitales. Guayaquil: Albatros. Recuperado de:

http://www.marcprensky.com/writing/Prensky-

NATIVOS\%20E\%20INMIGRANTES\%20DIGITALES\%20(SEK).pdf 
Riccetti, A. \& Rigo, D. (2018). Valoraciones acerca de una propuesta académica invertida en la formación de profesores en educación física. IV Congreso de educación física y deporte escolar. Villa Mercedes, San Luis, Argentina. Recuperado de: https://www.aacademica.org/iv.congreso.de.educacion.fisica.y.deporte.escolar

Rigo, D. \& Paoloni, P. (2017). Primera experiencia de clase invertida. Comprometer desde otro lugar. En Ruiz-Palmero, J., Sánchez-Rodríguez, J. y Sánchez-Rivas, E. (Edit.). Innovación docente y uso de las TIC en educación (pp.1-9). Málaga: UMA Editorial.

Ros Gálvez, A. \& Rosa García, A. (2014). Uso del vídeo docente para la clase invertida: evaluación, ventajas e inconvenientes. En B., Peña Acuña (Coord.). Vectores de la pedagogía docente actual (pp. 423-441). Madrid: ACCl.

Simon Llovet, J., Ojando Pons, E., Avila Morena, X., Miralpeix Bosch, A., Lopez Vicente, P. \& Prats Fernández, M. (2018). Reformulación de los roles del docente y del discente en la educación. El caso práctico del modelo de la Flipped Classroom en la universidad. Revista de Estudios y Experiencias en Educación, 2, 53-73. Recuperado de: http://www.rexe.cl/ojournal/index.php/rexe/article/view/491/407

Uzunboylu, H. \& Damla K. (2017). The Emerging Trend of the Flipped Classroom: A Content Analysis of Published Articles between 2010 and 2015. RED. Revista de Educación a Distancia, 54:1-13. Recuperado de:

http://www.redalyc.org/articulo.oa?id=54751771004

Vasilachis, I. (2007). Estrategias de investigación cualitativa. Buenos Aires: Gedisa 